BULLETIN OF THE

AMERICAN MATHEMATICAL SOCIETY

Volume 77, Number 4, July 1971

\title{
PERTURBATIONS OF THE UNILATERAL SHIFT
}

\author{
BY SUE-CHIN LIN $^{1}$
}

Communicated by Peter Lax, December 2, 1970

Introduction. The study of the unilateral shift on a Hilbert space has been one of the most important subjects in operator theory, for it provides many useful examples and counterexamples to all parts of Hilbert space theory (see Halmos [1]). The purpose of this note is to announce our results concerning perturbations and similarity of the unilateral shift in a slightly general setting. To be precise, let $S$ be an isometry on a separable Hilbert space $H$. We were able to show that $S+P$ is similar to $S$ for a large class $\Re$ of $S$-admissible bounded linear operators $P$ on $H$. To each $P \in \Re$, we constructed explicitly a nonsingular bounded linear operator $W$ on $H$, such that $S+P$ $=W S W^{-1}$. In particular, the unilateral shift $S$ on $l^{2}$ of squaresummable sequences, which sends $\left(x_{0}, x_{1}, x_{2}, \cdots\right)$ into $\left(0, x_{0}, x_{1}\right.$, $\left.x_{2}, \cdots\right)$ is similar to $S+\mu P$ for all infinite matrices $P=\left(p_{n m}\right)$ with $\sum\left|p_{n m}\right|<\infty(n, m=0,1,2, \cdots \infty)$ and all sufficiently small complex parameters $\mu$. This result becomes interesting when it is compared with that of [2], where $P=\left(p_{n m}\right)$ are required to be strictly lower-triangular and $p_{n+1, n} \neq-1$ for all $n$, in addition to the assumption that $\sum\left|p_{n m}\right|<\infty$.

I would like to thank Professor T. Kato of Berkeley for calling my attention to this problem.

1. $S$-admissible operators. Throughout this note, let $H, H^{\prime}$ be separable Hilbert spaces and $Y=l^{2}\left(0, \infty ; H^{\prime}\right)$. We denote by $\mathfrak{B}\left(H, H^{\prime}\right)$ the space of all bounded linear operators on $H$ to $H^{\prime}$. We write $\mathfrak{B}(H)$ for $\mathfrak{B}(H, H)$. The symbol $\langle$,$\rangle stands for the inner products in$ $H$ and $H^{\prime}$.

Definition 1.1. Let $S$ be an isometry on $H$ and $A \in \mathfrak{B}\left(H, H^{\prime}\right)$. The operator $A$ is said to be $S$-smooth, if there exists a constant $M$ $<\infty$ such that

$$
\sum_{n=0}^{\infty}\left\|A S^{n} u\right\|^{2} \leqq M^{2}\|u\|^{2} \text { and } \sum_{n=0}^{\infty}\left\|A S^{* n} u\right\|^{2} \leqq M^{2}\|u\|^{2},
$$

AMS 1969 subject classifications. Primary 4748.

Key words and phrases. Hilbert space, operator, perturbation, isometry, unilateral shift, similarity, nonsingular operator, infinite matrices.

${ }^{1}$ Research supported by NSF Grant GP-7952-X2 made to the Institute for Advanced Study. 
for all $u \in H$. In this case, we set $|A| s$ to be the infimum of all constants $M$ satisfying (1).

To each isometry $S$ on $H$ and operators $A, B \in \mathfrak{B}\left(H, H^{\prime}\right)$, we let

$$
D(A, B, S)=\left\{f \in Y: \sum_{n=1}^{\infty}\left\|\sum_{r=1}^{n} A S^{n-r} B^{*} f(r-1)\right\|^{2}<\infty\right\}
$$

and define

$$
\begin{array}{rlrl}
T(A, B, S) f(n)= & 0, & n & =0 ; \\
& =\sum_{r=1}^{n} A S^{n-r} B^{*} f(r-1), & n \geqq 1, \text { for } f \in D(A, B, S) .
\end{array}
$$

$T(A, B, S)$ is clearly a linear operator from $D(A, B, S)$ into $Y$. This operator $T(A, B, S)$ is a discrete version of the convolution operator $C$ defined in [3].

Definition 1.2. A class $\Re(S) \subset \mathfrak{B}(H)$ of operators is called a $S$ admissible class, if each $P \in \mathfrak{N}(S)$ can be written as the product $P$ $=B^{*} A$ of two operators $A, B \in \mathfrak{B}\left(H, H^{\prime}\right)$, satisfying the following properties:

(i) both $A$ and $B$ are $S$-smooth,

(ii) the operator $T(A, B, S)$ is a bounded linear operator on $Y$ into $Y$.

To each $P \in \Re(S)$, we set

$$
\llbracket P \rrbracket=\max \left(\|T(A, B, S)\|,|A|_{S} \cdot|B|_{S}\right),
$$

where $\|T(A, B, S)\|$ stands for its operator norm in $\mathfrak{B}(Y)$.

\section{The main results.}

THEOREM. Let $S$ be an isometry on $H$. Then for each $P=B^{*} A$ belongs to $\Re(S)$, and for any complex number $\mu$ with $|\mu|<1 / 2 \rrbracket P \rrbracket$, the operator $S+\mu P$ is similar to $S$. The operator $W(\mu)$, given by the following formula,

$$
\langle W(\mu) u, v\rangle=\langle u, v\rangle+\mu \sum_{n=0}^{\infty}\left\langle A S^{* n+1} u, B(S+\mu P)^{* n v}\right\rangle, \quad u, v \in H,
$$

is a nonsingular bounded linear operator on $H$ which implements the similarity, i.e. we have $(S+\mu P) W(\mu)=W(\mu) S$.

Example. Let $H=l^{2}(0, \infty), S=$ the unilateral shift on $H$ and $P$ $=\left(p_{n m}\right)$ be an infinite matrix with $|P|=\sum_{n, m=0}^{\infty}\left|p_{n m}\right|<\infty$. Let $H^{\prime}=l^{2}((0, \infty) \times(0, \infty))$. Define $A, B$ from $H$ to $H^{\prime}$ as follows: 


$$
A x_{n m}=\left|p_{n m}\right|^{1 / 2} x_{m},
$$

and

$$
B x_{n m}=\alpha_{n m}\left|p_{n m}\right|^{1 / 2} x_{n}, \text { for all } x \in l^{2}(0, \infty),
$$

where

$$
\alpha_{n m}=p_{n m} /\left|p_{n m}\right| \quad \text { for } p_{n m} \neq 0 \text { and } \alpha_{n m}=0 \text { when } p_{n m}=0 \text {. }
$$

It can be verified easily that $A, B \in \mathfrak{B}\left(H, H^{\prime}\right)$ with $B^{*} A=P$. We were able to show that $A, B$ are $S$-smooth with $|A| s$ and $|B| s$ both bounded by $|P|^{1 / 2}$. And the operator $T(A, B, S)$ belongs to $\mathfrak{B}(Y)$ with its norm bounded by $|P|$. Consequently, every infinite matrix $P=\left(p_{n m}\right)$ with $|P|=\sum_{n, m=0}^{\infty}\left|p_{n m}\right|<\infty$ is $S$-admissible with $\llbracket P \rrbracket$ $\leqq|P|$. We therefore have the following:

Corollary. Let $S$ be the unilateral shift on $l^{2}(0, \infty)$. If $P=\left(p_{n m}\right)$ is an infinite matrix with $|P|=\sum_{n, m=0}^{\infty}\left|p_{n m}\right|<\infty$, then for all complex numbers $\mu$ with $|\mu|<1 / 2|p|, S+\mu P$ and $S$ are similar operators on $l^{2}(0, \infty)$.

3. Outline of proof. To each $S$-admissible operator $P=B^{*} A$, we need to prove that there exists a constant $K<\infty$ such that

$$
\sum_{n=0}^{\infty}\left\|B(S+\mu P)^{* n} u\right\|^{2} \leqq K\|u\|^{2}, \quad \text { for all } u \in H .
$$

This is the main and the most difficult part of the entire proof. There seems to be no easy way to get a reasonable estimate for $\left\|B(S+\mu P)^{*} u\right\|$, and moreover $S, P$ do not necessarily commute. To get around these difficulties, we first constructed a family $U(n, \mu)$ $\in \mathfrak{B}(H)(n=0,1,2, \cdots)$ having the property

$$
\left(\sum_{n=0}^{\infty}\left\|B U(n, \mu)^{*} u\right\|^{2}\right)^{1 / 2} \leqq|B|_{S}\left(1-|\mu|[P \llbracket)^{-1}\|u\| .\right.
$$

Next we show that $(S+\mu P) U(n, \mu)=U(n+1, \mu)$ for all $n$. It follows that $U(n, \mu)=(S+\mu P)^{n}$, hence the inequality (3). Once the inequality (3) is obtained, we write $W(\mu)=1+\mu Q(\mu)$, with

$$
\|Q(\mu)\| \leqq|A|_{S}|B|_{S}(1-|\mu| \rrbracket P \rrbracket)^{-1} .
$$

Nonsingularity of $W(\mu)$ follows when we chose $\mu$ sufficiently small to make $\|\mu Q(\mu)\|<1$. Finally $(S+\mu P) W(\mu)=W(\mu) S$ is established by a routine manipulation and observing that $S^{*} S=1$. 
REMARK. If $S$ is unitary on $H$, then our Theorem holds for all parameters $\mu$ with $|\mu|<1 / \rrbracket P \rrbracket$ and we have, for $u, v \in H$,

$$
\left\langle W(\mu)^{-1} u, v\right\rangle=\langle u, v\rangle+\mu \sum_{n=0}^{\infty}\left\langle A U(n, \mu) u, B S^{n+1} v\right\rangle .
$$

Generalization to $l^{p}(0, \infty)$ for perturbations of the unilateral shift $S$, together with full details for the above results will appear elsewhere.

\section{REFERENCES}

1. P. R. Halmos, A Hilbert space problem book, Van Nostrand, Princeton, N. J., 1967. MR $34 \# 8178$.

2. J. M. Freeman, Perturbations of the shift operator, Trans. Amer. Math. Soc. 114 (1965), 251-260. MR 30 \#2342.

3. S. C. Lin, Wave operators and similarity for generators of semigroups in Banach spaces, Trans. Amer. Math. Soc. 139 (1969), 469-494. MR 39 \#2013.

Institute for Advanced Study, Princeton, New Jersey 08540 